\title{
Maladie de Fukuyama ou un rétrotransposon ancestral au Pays du Soleil Levant
}

Les progrès considérables réalisés depuis la découverte de la dystrophine dans le domaine des myopathies ont permis de connaître la plupart des éléments du complexe protéique protégeant la cellule musculaire au cours de la contraction et de l'étirement musculaire. Aussi, les recherches sur la dystrophie musculaire congénitale de Fukuyama (FCMD) - maladie récessive à l'incidence élevée au japon (1/10 000 naissances) mais fort rare dans les autres pays du monde - semblaient une affaire nippone ne devant pas révolutionner la planète. On se trompait. La découverte du gène codant pour la fukutine ouvre de passionnantes perspectives non seulement dans l'histoire des populations en Extrême-Orient, mais sur le rôle des rétrotransposons dans les mutations humaines, et sur celui de certaines protéines sécrétées sur la fonction musculaire et la migration neuronale.

\section{La FCMD au Japon}

La maladie de Fukuyama se manifeste par une hypotonie précoce, mais comporte aussi une micropolygyrie (ou lissencéphalie de type II), diminution de taille des circonvolutions cérébrales due probablement à un trouble de la migration neuronale. Dans cette myopathie congénitale récessive autosomique, des études sur des familles japonaises permirent tout d'abord de situer le locus en 9q31 (m/s $\left.1994 n^{\circ} 1, p .122\right)$, puis d'observer un net déséquilibre de liaison des marqueurs polymorphes de la région candidate (le même haplotype dans plus de $80 \%$ des chromosomes FCMD), consé- quence d'un effet fondateur [1]. D'après les études de populations, l'ancêtre commun remonte à environ 2500 ans. Or, c'est précisément vers cette période qu'un peuplement venant de Chine et de Corée vint au Japon, les Yayoi qui apportaient avec eux de nouveaux savoirs, dont la métallurgie du bronze et du fer, et surtout la culture du riz par irrigation [2]. La mutation FCMD fut-elle apportée par l'un d'entre eux ou survint-elle chez un des indigènes, les Jomon, qui préexistaient dans l'île ? Par analyse des ADN des descendants de ces populations, il doit être possible de le savoir.

\section{La mutation japonaise}

La mutation fondatrice est extrêmement intéressante: il s'agit en effet d'une insertion d'un rétrotransposon, fixé de façon stable depuis plus de deux millénaires dans le gène $F C M D$ et directement responsable de la maladie. Le gène $F C M D$, actuellement cloné et séquencé s'étend sur $100 \mathrm{~kb}$ environ, avec 10 exons dont le second contient le codon d'initiation. Les niveaux les plus élevés d'expression normale des transcrits sont observés dans le cerveau, le muscle squelettique, le cœur et le pancréas. Le fragment inséré se trouve dans la région 3' non codante. Il est constitué de 27 copies d'une séquence répétée en tandem de type SINE (short interspersed sequence) flanquée de part et d'autre d'une séquence cible dupliquée de deux courtes répétitions directes, structure caractéristique d'un phénomène de rétrotransposition [3]. Chez les sujets homozygotes pour cette mutation insertionnelle, le transcrit ne peut être décelé lors de l'analyse des ARN messagers (Northern blot). Dans la population japonaise, une personne sur 88 en est porteuse à l'état hétérozygote, ce qui correspond à la fréquence calculée des hétérozygotes FCMD au Japon. Mais, parmi les malades, on trouve aussi quelques hétérozygotes composites porteurs, sur l'autre allèle, de mutations ponctuelles ou délétionnelles qui n'entraînent pas la disparition complète du transcrit mais une diminution nette par rapport aux témoins. Il est donc probable que les mutations entraînent une diminution de la transcription et/ou une instabilité de l'ARNm avec perte de fonction. La mutation par délétion (avec codon stop prématuré) a été héritée d'un père d'origine anglo-saxonne. Il doit donc sans doute exister, très rarement, d'autres mutations FCMD dans le monde, donnant d'ailleurs éventuellement une symptomatologie un peu différente.

\section{La fukutine}

L'analyse séquentielle du gène $F C M D$ montre qu'il code pour une protéine, nommée fukutine, ne présentant pas d'homologie avec les protéines déjà répertoriées dans les banques de données mais contenant une séquence signal dans la région amino-terminale, avec un site de clivage présumé au codon 21 . L'hypothèse d'une protéine sécrétée est confirmée par des études en cellules COS-7: transfectées avec un gène codant pour une protéine de fusion fukutine-protéine fluorescente verte ou fukutine-hémagglutinine, le signal intracellulaire apparaît dans l'appareil de Golgi pour diffuser dans le 
cytoplasme puis la fukutine est emmagasinée dans des vésicules sécrétoires. La diminution de la $\beta$-dystroglycane et de la laminine $\alpha-2$, ligand de l' $\alpha$-dystroglycane, chez les malades atteints de FCMD, pourrait orienter vers une interaction de la fukutine, située dans la matrice extracellulaire, avec le complexe dystrophine-glycoprotéine.

\section{Fukutine et migration neuronale}

Dans la migration neuronale et l'histogenèse du cortex cérébral, quatre gènes principaux ont été récemment décrits : RELN pour reeler et disabled, découverts grâce à des mutations chez la souris [4], LIS1 ou $P A F A H 1 B 1$ pour acétylhydrolase $\beta 1$ du PAF (platelet activating factor) dans le syndrome de Miller-Dieker $(\mathrm{m} / \mathrm{s}$ $\left.1998 n^{\circ} 11, p .1259\right)$ et DOUBLE-CORTINE (DCX) enfin dans les hétérotopies laminaires sous corticales (lissencéphalie liée à l'X [5], comme la reelin, la fukutine est une glycoprotéine sécrétée. Elle pourrait donc interagir avec elle sur les neurones de la plaque corticale et/ou sur l'écheveau des fibres gliales qui guident la migration neuronale, à moins qu'elle n'intervienne directement, dans le cerveau et dans le muscle, sur la structure de la lame basale. En effet, de nettes anomalies de celle-ci sont visibles au microscope électronique dans la FCMD.

Quoi qu'il en soit, il existe deux autres myopathies congénitales comportant aussi des troubles de migration neuronale : le syndrome de Walker-Warburg et la maladie muscle-œil-cerveau, observée en Finlande, dont les manifestations cliniques voisines incitent à vérifier au plus vite l'intégrité du gène FCMD [6].

Enfin, si cette découverte nippone a des implications diagnostiques immédiates pour la population japonaise, elle ouvre aussi des perspectives de thérapie génique: on pourrait en effet imaginer des moyens de contrer les effets de l'insertion, en augmentant par exemple la stabilité de l'ARNm fukutine-rétrotransposon. La difficulté majeure d'une approche thérapeutique de cette affection dont les manifestations sont déjà néonatales, résidera probablement dans le choix du moment de son intervention.

H.G.

1. Toda T, Miyake M, Kobayashi K, Mizuno K, Saito $\mathrm{K}$, et al. Linkage-disequilibrium mapping narrows the Fukuyama-type congenital muscular dystrophy (FCMD) candidate region to less than 100 kb. Am J Hum Genet 1996 ; 59 : 1313-20.

2. Eberhart DE, Curran T. Yayoi era mutation disrupts brain and muscle. Nat Med 1998 ; 9 : 1002-3. 3. Kobayashi K, Nakahori Y, Miyake M, Matsumura K, Kondo-Iida E, et al. An ancient retrotransposal insertion causes Fukuyama-type congenital muscular dystrophy. Nature 1998 ; 394 : 388-92.

4. Goffinet A. Mutation reeler et protéine reelin : bases d'un nouveau modèle du développement cortical. Med Sci 1996 ; 12 : 631-5.

5. Des Portes V, Pinard J, Chelly J. Doublecortine, un nouveau gène exprimé dans le cerveau fotal, est impliqué dans les hétérotopies laminaires sous-corticales et les lissencéphalies liées au chromosome X. Med Sci 1998 ; 14 : 241.

6. Voit T. Congenital muscular dystrophies: 1997 update. Brain Dev $1998 ; 20$ : 65-74.

\title{
SOCIÉTÉ DE BIOLOGIE
}

Cent-cinquantenaire de la Société de Biologie

\section{9 novembre 1998 (14 h - 18 h)}

\section{Séance des membres correspondants de la Société de Biologie}

\author{
Harold Kalant (Professeur à I'Université de Toronto) \\ Rôle de la Science dans la formulation de la politique sociale sur la drogue \\ Christian Doutremépuich (Professeur à I'Université de Bordeaux) \\ Polymorphisme de I'ADN, applications médico-légales \\ Ba Denian (Président de l'Académie des Sciences Médicales de Chine) \\ La recherche en immunologie du cancer en Chine \\ Chen Zhu (Directeur de I'Institut d'Hématologie de Shanghai II) \\ Le génome humain en Chine \\ Susan Hollán (Professeur à I'Institut National d'Hématologie et d'Immunologie de Budapest) \\ Déficiences en enzymes glycolytiques et neurodégénérescence \\ Gustav Born (Professeur à I'Institut de Recherche William-Harvey de Londres)
}

Les effets d'hormones et de médicaments sur la fixation de protéines plasmatiques athérogènes sur les parois artérielles

Hannes Stähelin (Professeur à I'Hôpital Cantonal de I'Université de Bâle)

Vitamine $D$ plasmatique et force musculaire chez les sujets âgés

Francis Karst (Professeur à I'Université de Poitiers)

Biosynthèse et transport des stérols chez la levure

Collège de France

11, place Marcelin-Berthelot -75005 Paris, France 